\title{
An Innovative Space Rover with Extended Climbing Abilities

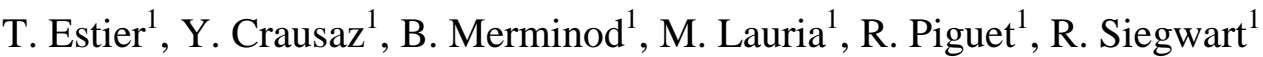

\section{Abstract}

Autonomous mobile robots have become a key technology for unmanned planetary missions. To cope with the rough terrain encountered on most of the planets of interest, new locomotion concepts for rovers and micro-rovers have to be developed and investigated. The most advanced locomotion concepts are based on wheels or caterpillars (e.g. Sojourner (NASA) or Nanokhod (ESA)). These rovers have clear advantages regarding power efficiency and complexity if compared with walking robots. However, they still have quite limited climbing abilities. Typically they can only overcome obstacle of their wheel size.

In our paper we present an innovative rover concept with 6 motorized wheels. Using a rhombus configuration, the rover has a steering wheel in the front and the rear, and two wheels arranged on a bogie on each side. The front wheel has a spring suspension to guarantee optimal ground contact of all wheels at any time. The steering of the rover is realized by synchronizing the steering of the front and rear wheels and the speed difference of the bogie wheels. This allows for high precision maneuvers and even turning on the spot with minimum slip. The use of parallel articulations for the front wheel and the bogies enables to set a virtual center of rotation at the level of the wheel axis. This insures maximum stability and climbing abilities even for very low friction coefficients between the wheel and the ground.

A well functioning prototype has been designed and manufactured in our lab. It shows excellent performance surpassing our expectations. This rover is able to passively overcome unstructured obstacles of up to two times its wheel diameter and can climb stairs with steps of over $20 \mathrm{~cm}$.

\section{Introduction}

Planetary exploration rovers need to show strong off-road capabilities due to the unstructured environment met during their mission. With the new millennium, the nature of the planetary missions will change from pure exploration to real exploitation. That means long-range missions, permanent constructions and humanrobots cohabitation. This will drastically modify the nature of the operational environment which will be both structured and unstructured, especially in the case of construction robotics.

Most of the existing surface locomotion concepts are based on wheels, caterpillars or legs rovers.

\footnotetext{
${ }^{1}$ Institute of Robotic Systems, EPFL (Swiss Federal Institute of Technology Lausanne), Switzerland
} 
-Walking machines (e.g. Dante [BAR97]) are well adapted to unstructured environment because they can insure their stability in a wide range of situations, but they are mechanically complex and require a lot of control resources. On a plane surface, they demonstrate low speed motion and high power consumption if compared with the other solutions.

- Caterpillars vehicles demonstrate good off-road abilities because of their stability and good friction coefficient during motion. Its advantage is the simplicity but the friction losses between the surface and the caterpillars when the robot is turning are high. The Nanokhod [WIN99], a robot based on this locomotion solution, is planed to be send on Mars by the ESA. It is able to recover after a flip-over using its central payload arm. But on the other hand the failure of one caterpillar motor stops the mobility of the robot.

- Wheeled rovers are the optimal solutions for well structured environment like roads or habitations. But off-the road, their efficiency is very dependent on the typical size of encountered obstacles that have to be overcome in a standard motion mode. This is the case for Sojourner [STO96], its son Rocky 7 [VOL97] or Micro5 [KUB99], which can typically overcome obstacles of their wheel size, if friction is high enough. Adding real climbing abilities to a wheeled rover requires the use of a special strategy and often implies dedicated actuators like for the Marsokhod [KEM92] and Hybtor [LEP98] or complex control procedure like for the SpaceCat [LAU98] or for the Nanorover [TUN99].

The classification we use in our lab to study locomotion concepts makes the difference between active and passive locomotion. Passive locomotion is based on passive suspensions, that means no sensors or additional actuators to guarantee stable movement. On the opposite, an active robot implies a close control loop to keep the stability of the system during motion. Under these definitions Sojourner, Rocky 7 and Micro5 are passive robots; walking machines, Nanorover [TUN99] and SpaceCat are active robots; Marsokhod, Nanokhod and Hybtor are hybrid depending on their locomotion mode.

It is clear that active locomotion extends the mobility of a robot but increases the complexity and needs extended control resources. With the actual speed of the controllers, it is yet imaginable to perform active locomotion and this is one of our research axes. However, in the case of space autonomous robotics, predominant criteria are certainly the power consumption, the reliability and the mobility of the system. High complexity of active robots and the poor climbing abilities of passive systems motivated us to develop and investigate new locomotion concepts for passive and wheeled rovers.

These considerations were the bases for an undergraduate project whose goal was to conceive and build a mobile robot based on the following requirements: a) wheeled rover showing good off-road abilities: maximum gripping capacity and stability during motion even in rough terrain b) passive overcoming of steps of 1,5 times its wheel diameter.

The result of this project is a first prototype manufactured in our lab that shows performance surpassing our expectations. The presentation of this prototype and its test results are the subject of this article. 


\section{Mechanical Robot Design}

\subsection{Overview}

Using a rhombus configuration (c.f. fig. 7), the rover has one wheel mounted on a fork in the front, one wheel in the rear and two bogies on each side. Although our bogies have a

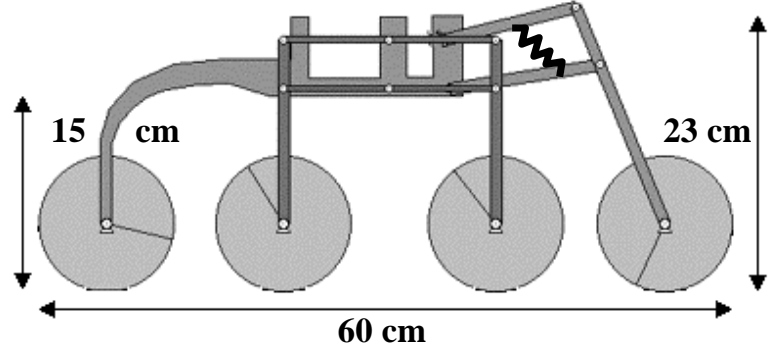

Figure 1 : Schematic lateral view of the rover special geometry, it is the same basic principle as used for a train suspension : a couple of two wheels mounted on a support which can freely rotate around a central pivot.

The front fork has two roles : its spring suspension guarantees optimal ground contact of all wheels at any time and its particular parallel mechanism produce an elevation of the front wheel if an obstacle is encountered (fig. 2).

The parallel architecture of the bogies and the spring suspended fork provides a non-hyperstatic configuration for the 6 motori-

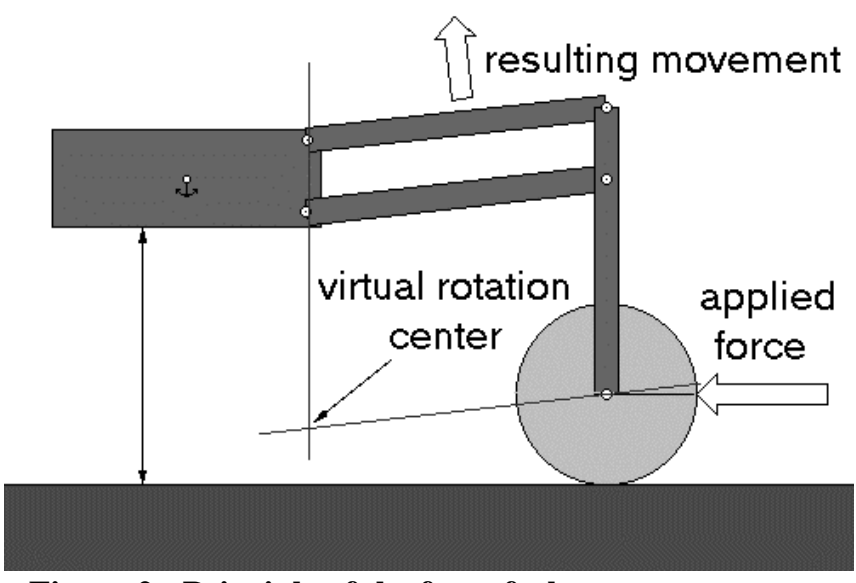

Figure 2 : Principle of the front fork zed wheels while maintaining a high ground clearance. This insures maximum stability and adaptability as well as excellent climbing abilities. The rover is designed to keep all its 6 motorized wheels in contact with the ground on a convex ground up to a minimal radius of $30 \mathrm{~cm}$ and on a concave ground up to a minimal radius of 35 $\mathrm{cm}$.
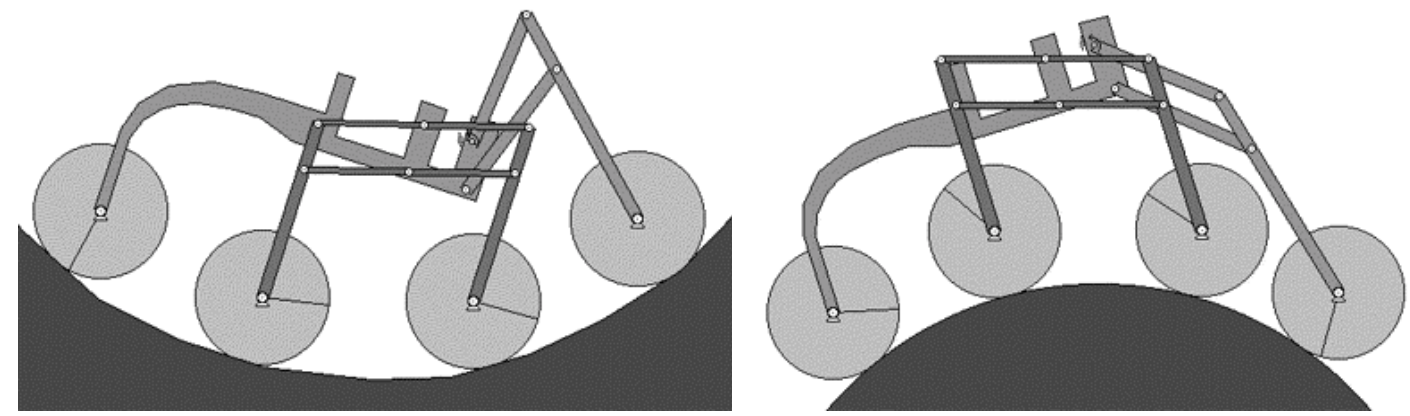

Figure 3 : Rover in a concave and convex ground

The steering system (explained later in this article) allows the rover to carry out a pure rotation even in these extreme situations. The six wheels are motorized by motors of $1.75 \mathrm{~W}$ driven with constant PWM. We use IR remote control to set the speed of the rover. The total weight is $3.1 \mathrm{~kg}$ including $600 \mathrm{~g}$ for the battery (12 $\mathrm{V} / 2000 \mathrm{mhA}$ ). 


\subsection{The Bogies}

The bogies are the first key components of the rover. They provide the lateral stability during the motion even on very rough terrain. To insure good adaptability of the bogie, it is necessary to set the pivot as low

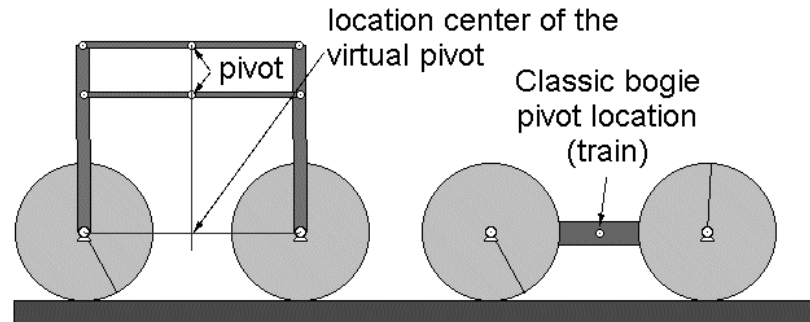

Figure 4 : explaination of the bogie architecture as possible and in the same time to keep a maximum ground clearance. This problem is solved by using the parallel configuration showed on fig. 4 that bring the virtual center of rotation of the bogie at the height of the wheel axis.

\subsection{The Front Fork}

As shown on Fig. 2, a trajectory of the front wheel with an instantaneous center of rotation situated under the wheel axis is helpful to get on an obstacle. The second goal for the fork is to provide a maximum vertical amplitude for the wheel. To find the optimal configuration for the fork, we established the following kinematic model (fig. 5) :

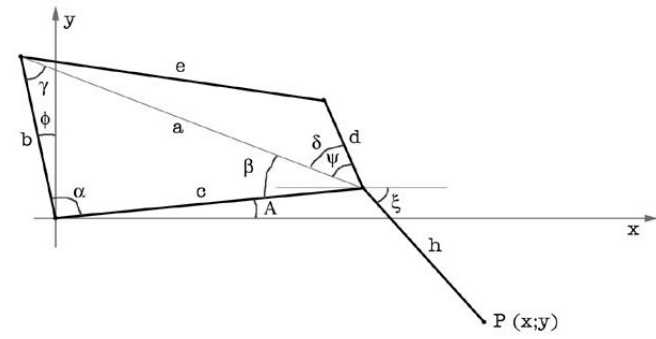

Figure 5 : a) parametric model of the fork

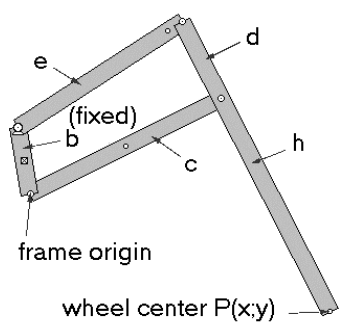

b) final configuration

With the parametric equations of $\xi$, $\alpha$ et $\psi$ as function of the angle $A$,

- $\alpha(A)=\frac{\pi}{2}-A+\phi$

- $\psi(A)=a \cos \left[\frac{c-b \cdot \cos [\alpha(A)]}{\left.\sqrt{b^{2}+c^{2}-2 \cdot b \cdot c \cdot \cos [\alpha(A)}\right]}\right]+a \cos \left[\frac{b^{2}+c^{2}-2 \cdot b \cdot c \cdot \cos [\alpha(A)]+d^{2}-e^{2}}{\left.2 \cdot d \cdot \sqrt{b^{2}+c^{2}-2 \cdot b \cdot c \cdot \cos [\alpha(A)}\right]}\right.$

- $\xi(A)=A-\psi(A)$

we have all elements to establish the position of the point $P$ as function of the angle $A$ :

$$
P(A)=\left(\begin{array}{l}
c \cdot \cos (A)+h \cdot \cos [\xi(A)]) \\
c \cdot \sin (A)+h \cdot \sin [\xi(A)]
\end{array}\right.
$$

Finally, we chose the different parameters to get the trajectory shown on fig. 6. The horizontal line is the height of the wheel axis when the robot is on a horizontal plane. Note that the characteristic of the trajectory under this line is needed to insure a good stability when the rover is on a convex ground.

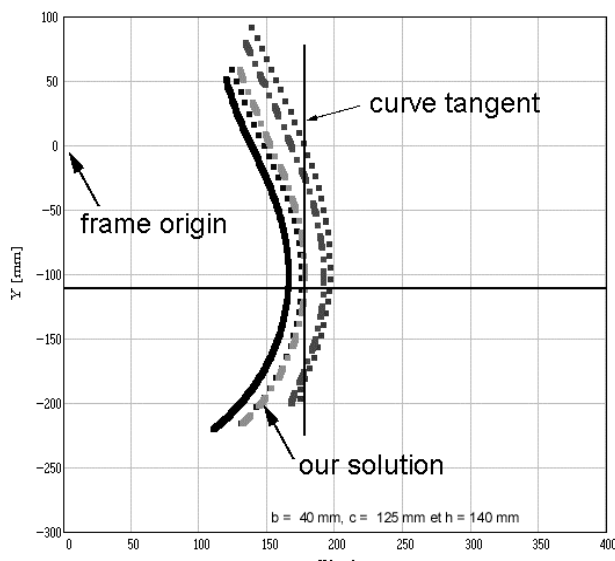

Figure $6: P(A)$ trajectories for different value of the segments $e$ and $d$ 


\subsection{Steering}

The steering of the rover is realized by synchronizing the steering of the front and rear wheels and the speed difference of the bogie wheels. This allows for high precision maneuvers and even turning on the spot with minimum slip.

\section{Experimental Results}

\subsection{Motion in structured environment}

\subsubsection{Step}

One of the requirements of this rover was the overcoming of

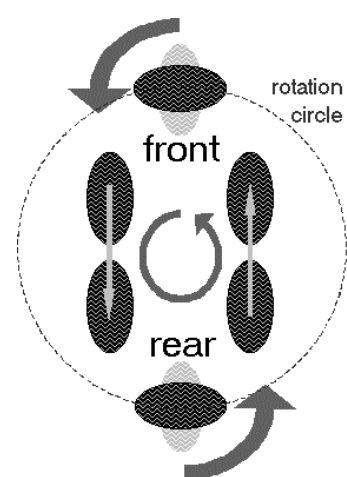

Figure 7 : Configuration of the wheels on ground a step which height had to be at least 1.5 times the wheel diameter. The fig. 8 shows the main sequences of the rover climbing a step. First, the front fork gets on the step, compressing its spring (shown fig. 1), then the energy accumulated in the spring helps the first wheel of the bogie to climb. When the second bogie wheel is in contact with the wall, the bogie turns around the step. At this time the center of gravity reached almost its final height. Finally, the last wheel can easily get on the step.

a)

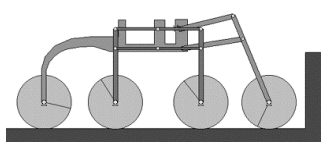

\begin{abstract}
b)
\end{abstract}

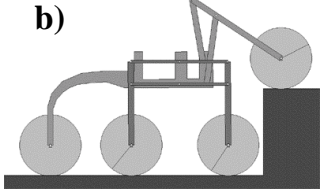

c)

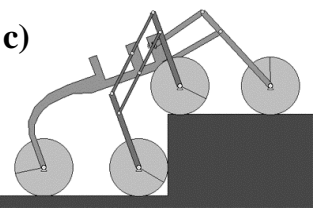

d)

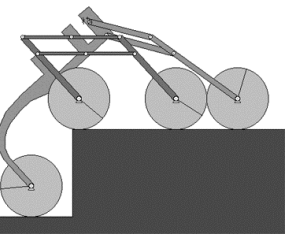

Figure 8 : Sequences showing the rover climbing a step (height is 1.5 times the wheel diameter) As the two bogies are independent from each other, it is even possible to climb the step if the robot is not approaching perpendicularly or if only one bogie encounters a step. Although it was designed to climb steps up to $17 \mathrm{~cm}$, the rover is able to climb even steps of twice its wheel diameter $(22 \mathrm{~cm})$.
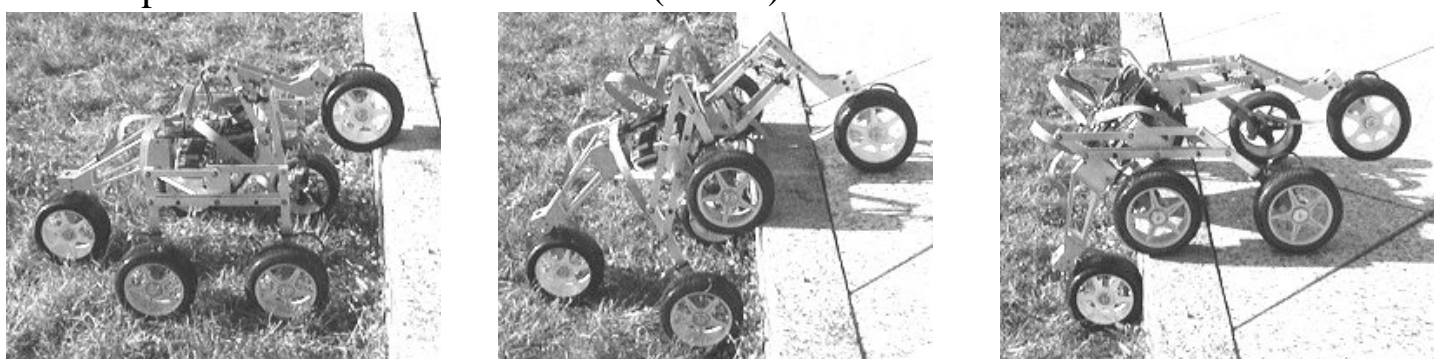

Figure 9 : Climbing sequence for a step of $22 \mathrm{~cm}$ high ( 2 times the wheel diameter)

\subsubsection{Stairs}

Impressed by its step climbing abilities, we tested the rover also for stairs climbing. Due to a good correlation between the bogies size and the steps dimensions, the rover is able to climb them easily.

This is impressive if it is considered that the $1,75 \mathrm{~W}$ motors are controlled in open loop.

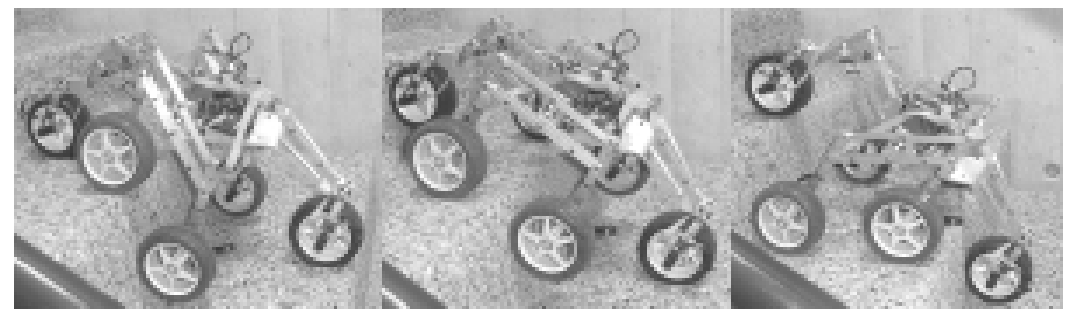

Figure 10 : Climbing sequence in stairs $(h=17 \mathrm{~cm}, w=21 \mathrm{~cm})$ 


\subsection{Unstructured Environments}

3.2.1. Off-road Abilities

The rover demonstrates excellent stability in both smooth and rough terrain. It still moves with a lateral or frontal inclination of $40^{\circ}$ (fig. 11 $\mathrm{a}, \mathrm{b}, \mathrm{c})$ and is able to overcome obstacles like rocks even with a single bogie (fig.11 b, d, e).

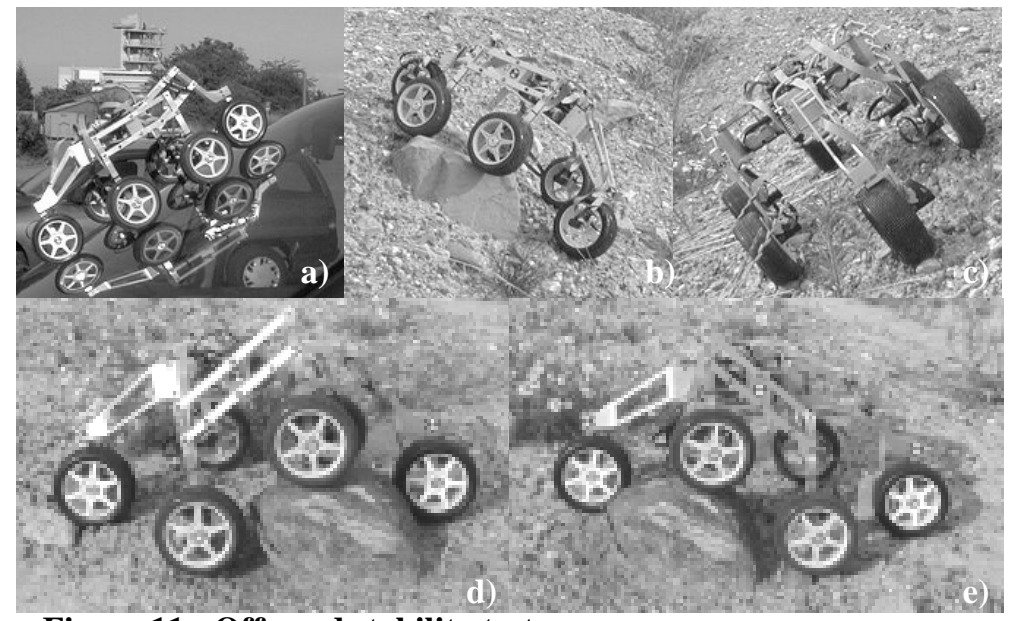

Figure 11 : Off-road stability tests

The rover was tested in various terrains (sandy and gravelly soil) and showed that its architecture was well adapted for fields motion even in dunes or in furrows.

\subsubsection{Mean Free Path}

Using the models described in [WIL97], we computed the Mean Free Path (MFP) for the two Viking landing sites (VL1 and VL2). We set the height of passively climbable obstacles to $17 \mathrm{~cm}$ instead of the true value of twice its wheel diameter because there is at this time no payload on the rover. For VL1, we obtained a value of 35.7 (Sojourner: 9.6). For VL2, the MFP is 5.4 (Sojourner: 2.4). This is a clue to say that this rover would be a good candidate for long range missions.

\subsection{Movement of the CoG}

The climbing ability is mostly given by the sequential rising of the center of gravity $(\mathrm{CoG})$ provided by the consecutive action of the wheels.

Figure 12 shows the trajectory of $\mathrm{CoG}$ for a step climbing of $17 \mathrm{~cm}$. The center of gravity goes up to $10 \%$ of the final height when the front wheel is on the top of the step (fig 12b). Then the first bogie

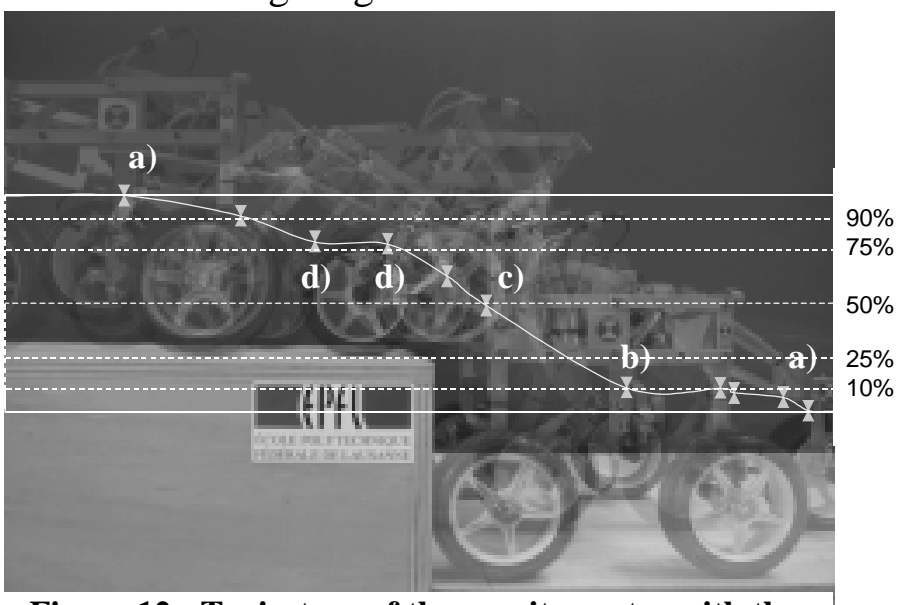

Figure 12 : Trajectory of the gravity center with the corresponding configurations defined in fig. 8 wheel, helped by the action of the front fork, brings the $\mathrm{CoG}$ to $50 \%$ (fig. $12 \mathrm{c}$ ). The second bogie wheel and the rear wheel contribute each for approximately $25 \%$. It can be seen that the mechanical structure allows a smooth movement of the CoG.

\subsection{Influence of the Friction Coefficient}

To show that the friction between the wheels and the ground are less critical with this architecture than with classical rovers, we reduced the friction coefficient of some wheels by covering the tire with a plastic film tape. The measured static friction coefficient between the uncovered wheels and the wood step is 0.81 and goes down 
to 0.23 with the plastic coverage. The rover was able to climb easily the wood step with the front and the rear wheel covered (bogies uncovered) or with the bogies wheels covered (front and rear wheel uncovered).

As we expected, the rover was not able to climb the step anymore with all wheels covered by plastic film. Nevertheless there is a large number of parameters which are not optimized on this first prototype like the weight or the control of the motors.

\section{Conclusion}

In this paper we proposed an innovative wheeled rover which provides excellent climbing and steering capabilities. Based on a parallel architecture allowing high ground clearance and in the same time very good stability, this rover is able to passively overcome steps of twice its wheel diameter, to climb stairs or to move in very rough terrain. These capabilities are mainly provided by the parallel architecture of the suspension in combination with non-hyperstatic contact for all its wheels with the ground.

This rover is the perfect candidate for long range planetary missions as well as for operations in environment that are both structured and unstructured like for space construction robotics. Terrestrial applications are also numerous: indoor and outdoor surveillance, ventilating shaft cleaning, mining and construction machines, agriculture, post earthquake assistance or even mine clearance if good sensors appear.

In a next step, the robot will be equipped with adequate sensors for fully autonomous operations.

\section{References}

[BAR97] J. Bares, D. Wettergreen, "Lessons from the Development and Deployment of Dante II," Proceedings of the 1997 Field and Service Robotics Conference, December, 1997.

[KEM92]A. L. Kemurdjian, V. Gromov, V. Mishkinyuk, V. Kucherenko, P. Sologub, «Small Marsokhod Configuration», International Conference on Robotics \& Automation, Nice, 1992

[KUB99] T. Kubota, Y. Kuroda, Y. Kunii, I. Natakani, "Micro Planetary Rover 'Micro5' ", Proceedings of Fifth International Symposium on Artificial Intelligence, Robotics and Automation in Space (ESA SP-440), pp.373-378, Noordwijk, 1999.

[LAU98] Lauria M., Conti F., Maeusli P.-A., Van Winnendael M., Bertrand R., Siegwart R.: "Design and Control of an Innovative Micro-Rover", Proceedings of 5th ESA Workshop on Advanced Space Technologies for Robotics and Automation, The Netherlands, 1998.

[LEP98] I. Leppänen, S. Salmi, A. Halme, "WorkPartner HUT Automation's new hybrid walking machine", CLAWAR'98 First international symposium, Brussels, 1998.

[STO96] H. W. Stone, "Mars Pathfinder Microrover: A Low-Cost, Low-Power Spacecraft", Proceedings of the 1996 AIAA Forum on Advanced Developments in Space Robotics, Madison WI, 1996.

[TUN99] E. Tunstel "Evolution of Autonomous Self-Righting Behaviors for Articulated Nanrovers" Proceedings of Fifth International Symposium on Artificial Intelligence, Robotics and Automation in Space (ESA SP-440), pp.341-346, Noordwijk, 1999.

[VOL97] R. Volpe, J. Balaram, T. Ohm, R. Ivlev. "Rocky 7: A Next Generation Mars Rover Prototype." Journal of Advanced Robotics., 11(4), December 1997.

[WIL97] B. Wilcox, A. Nasif, R. Welch, "Implications of Martian Rock Distributions on Rover Scaling", Planetary Society International Conference on Mobile Robots and Rover Roundup, Santa Monica, 1997.

[WIN99] M. Van Winnendael, G. Visenti, R. Bertrand, R. Rieder, "Nanokhod Microrover Heading towards Mars", Proceedings of Fifth International Symposium on Artificial Intelligence, Robotics and Automation in Space (ESA SP-440), pp.69-76, Noordwijk, 1999. 